transfer the mildew resistance to other cultivars of the cultivated oat by a simple backcrossing programme.

TAING Aung

Hugh ThOMAs

Welsh Plant Breeding Station,

Plas Gogerddan, Aberystwyth SY23 3EB, UK

Received January 19; accepted February 25, 1976.

1 Lawes, D. A., and Hayes, J. D., Pl. Path., 14, 125 (1965).

2 Dyck, P. L., and Zillinsky, F. J., Can. J. Genet. Cytol., 5, 398 (1963).

3 Sadanaga, K., and Simons, M. D., Agron. J., 52, 285 (1960).

4 Gauthier, F. M., and McGinnis, R. C., Can.J. Genet. Cytol., 10, 186 (1968).

5 Thomas, H., and Bhatti, I. M., Euphytica, 24, 149 (1974).

Forsberg, R. A., and Nishiyama, 1., Crop Sci., 9, 723 (1969).

Sharma, D. C., Euphytica, 24, 503 (1975).

Dyck, P. L., and Rajhathy, T., Can.J. Genet. Cytol., 5, 408 (1963)

Thomas, H., Leggett, J. M., and Jones, I. T., Euphytica, 24, 717 (1975).

Driscoll, C. J., and Jensen, N. F., Genetics, 48, 459 (1963).

1 Rajhathy, T., Can. J. Genet. Cytol., 5, 127 (1963).

\section{Cyanogenic polymorphism in bracken in relation to herbivore predation}

THE ecological success of bracken is partly a result of its extensive rhizome system, and because of its ability to synthesise various secondary compounds which deter predators and phytopathogens ${ }^{1-3}$. These compounds include the cyanogenic glycoside, prunasin ${ }^{4}$, toxic because on enzymatichydrolysis $\mathrm{HCN}$ is released. Although present in only $5 \%$ of ferns ${ }^{5}$, cyanogenic glycosides are ecologically significant in other plant species; they deter both molluscs and voles from grazing on Lotus corniculatus $^{6}$, and rabbits from eating Trifolium repens ${ }^{7}$. As in these two species, we have found that cyanogenesis is also polymorphic in bracken.

Our aim was to correlate herbivore predation with changes in cyanogenesis and phenolic content of bracken throughout the growing season (G.A.C.-D., E. Bernays, S. Finch and T.S., unpublished). We examined bracken from several randomly chosen sites in Richmond Park, Surrey, These comprised open and shady areas, and involved both inter- and intra-population studies. Our results showed that in most populations $96 \%$ of individuals contained both prunasin and the corresponding hydrolase and were therefore cyanogenic, but in a few populations, $98 \%$ of individuals were acyanogenic. Of these, $85 \%$ lacked both enzyme and substrate, whereas $15 \%$ contained prunasin without enzyme. We found that the bracken fronds from such areas were heavily grazed by deer and sheep, as examination of animal droppings in the area and observations by the gamekeeper showed. Examination of other widely separated patches of bracken confirmed that only fronds from acyanogenic plants were eaten.

To establish whether it is the production of $\mathrm{HCN}$ or merely the possession of prunasin which is the predator deterrent, we fed disks of bracken fronds to the phytophagous locust, Schistocerca gregaria. The results of our experiments showed that when both prunasin and enzyme were present only $4 \%$ of fronds were eaten, whereas when only the substrate was present the insects consumed $52 \%$ of the test meal, which was nearly the same as when both substrate and enzyme were absent $(48 \%)$. It seems, therefore, that the production of HCN (or benzaldehyde) acts as the deterrent. Young fronds of bracken were more cyanogenic than older fronds, whether sampled throughout the season, or as young and spore-bearing fronds of a single plant. We found that $S$. gregaria consumed the minimum amount of bracken in test meals during late May to early June, coinciding with the greatest intensity of cyanogenesis".

We conclude that cyanogenic polymorphism in bracken has a positive role in determining the degree of herbivore predation and that there is selectivity for acyanogenic forms in natural conditions.

We thank Dr E. Bernays, C.O.P.R., Mr S. Finch, Brunel
University, and Mr Brown, gamekeeper of Richmond Park, for their assistance. This work was supported by the ARC.

G. A. COOper-Driver

T. SWAIN

ARC Biochemical Laboratory, Royal Botanic Gardens, Kew, Richmond, Surrey, UK

Received January 21; accepted February 26, 1976.

Cooper-Driver, G. A., Bot. J. Linn. Soc. (in the press). Lawton, J., ibid. (in the press).

Whittaker, R. H., and Feeny, P. P., Science, 171, 757-770 (1971)

Kofod, H., and Eyjolfsson, R., Tetrahedron Lett., 1289 (1966).

Eyjolfsson, R., thesis, Danmarks farmaceutiske Hoiskole, Copenhagen (1968). Jones, D. A., in Taxonomy and Ecology (edit. by Heywood, V. H.), 213-242 (Academic, London, 1973).

Corkill, L., N.Z.Jl Sci. Technol. A., 34, 1-16 (1952).

8 Moon, F. E., and Raafat, M. A., J. Sci. Fd Agric., 2, 327-336 (1951).

\section{Inhibitory binocular interaction in human vision and a possible mechanism subserving stereoscopic fusion}

IN the human visual system, pathways from the two eyes converge anatomically by partial crossing over of the optic nerve fibres at the optic chiasma. Neurophysiological studies of the primary visual cortex of primates have established that these converging pathways carry signals from both eyes to the same cortical neurones ${ }^{1}$. In normal viewing conditions, each eye forms an image of the external visual field, the point of attention in the field being centrally fixated in both eyes. There is a small difference in the relative positions of non-central image points formed on the two retinae because the eyes view the visual field from slightly different positions. The ability of the primate visual system to extract information about the depth of a focused object in the visual field apparently rests on the convergence on to single cortical neurones of signals arising from the slightly displaced images in the two eyes ${ }^{2}$. If each eye perceives a totally different visual stimulus, however, the visual signals arising from the two stimuli are perceptually antagonistic and, in this situation, an observer usually detects only one stimulus at a time. This phenomenon is referred to as binocular rivalry. We have investigated interactions between visual signals produced by two different stimuli when presented one to each eye.

Our studies concerned a special class of stimulus patternsone-dimensionally periodic distributions of light which, because of their alternating light and dark bar structure, are called grating stimuli (Fig. 1 inset). The visual detectability of such grating stimuli is frequently specified by the minimum (or threshold) value of contrast level at which the grating can just be detected, with the contrast defined as $\left(I_{\max }-I_{\min }\right) /\left(I_{\max }+I_{\min }\right)$, $I_{\max }$ and $I_{\mathrm{min}}$ being respectively the maximum and minimum illumination levels present in the grating stimulus pattern. It has been shown that the threshold contrast level for detection of a grating increases transiently after adaptation to a similar grating of high contrast ${ }^{3-5}$. This adaptation effect is observed only when the test and adaptation gratings are of similar spatial frequency (that is periodicity) and when they are oriented in near parallel directions. The adaptation effect can be studied by presenting both test and adaptation gratings to the same eye ${ }^{5}$, or by presenting them one to each eye $\mathrm{e}^{6}$, and both methods yield essentially the same results. We measured the grating adaptation effect with the test and adaptation gratings, matched in orientation and spatial frequency, presented to one eye and a further grating stimulus, which we call the conditioning grating, presented to the other eye (Fig. 1 inset). The results show that when the conditioning grating is of spatial frequency, $f_{\mathrm{cg}}$, in the approximate range $1-4$ cycles per degree, it suppresses 